\title{
Theory for Nonlinear Mie-Scattering from Spherical Metal Clusters
}

\author{
J. Dewitz, W. Hübner, and K. H. Bennemann \\ Institut für Theoretische Physik der Freien Universität Berlin,Arnimallee 14, 14195 Berlin, \\ Germany.
}

(September 30,1994)

\begin{abstract}
Using classical electrodynamics we determine the angular dependence of the light intensities radiated in second and third harmonic generation by spherical metal clusters. Forward and backward scattering is analyzed in detail. Also resonance effects in the integrated intensities are studied. Our work treats the case of intermediate cluster sizes. Thus it completes the scattering theory fo spherical clusters between Rayleigh-type analysis for small spheres and geometrical optics for spheres much larger than the wavelength for nonlinear optics. Since the particle size sensitivity of Mie-scattering is increased by nonlinearity, the results can be used to extract sizes of small particles from nonlinear optics.
\end{abstract}

61.46.+w; 42.65.Ky; 78.90.+t; 73.20.Mf. 


\section{INTRODUCTION}

In this paper we investigate the nonlinear interaction of light with small metal particles. In nonlinear optics one expects a more pronounced sensitivity of the radiated yield to the light-wavelength and cluster-size, due to effects by nonlinear sources.

The linear interaction of light with spherical objects shows certain changes depending on the size of the sphere. The limiting cases are Rayleigh- scattering for spheres small compared with the wavelength and reflection by spheres much larger than the wavelength (the limit of geometrical optics). If the size of the sphere and the wavelength of light have comparable magnitudes the intensities scattered by spherical metal clusters are strongly enhanced. Mie [1] first calculated this classical effect and found that most of the incident light intensity is scattered in the forward direction, because of interference of waves originating from the front and the back of the sphere. This so called Mie-effect is increased with increasing sphere size. Bohren and Huffmann [2] pointed out that the extinction efficiency $Q_{\text {ext }}$ defined as the sum of scattering and absorption efficiencies $\left(Q_{e x t}=Q_{s c}+Q_{a b s}\right)$ as a function of the sphere-radius are characterized by the interference- and ripple structure. The interference structure gives rise to regular interferences of incident and forward scattered light dominating the envelope of $Q_{\text {ext }}$, see Fig. (11). Superimposed on this is sharp and highly irregular ripple structure originating from resonant electromagnetic surface modes of the sphere. In general, the resonances depend on the size parameter $k a$ comparing the radius of the sphere $a$ and the wavelength expressed by the absolute value of the wave vector $k$ of the incident light. Thus resonances also occur by varying the wavelength of the incident light. For small spheres only

one peak or resonance appears at $\omega_{p} / \sqrt{3}$ with $\omega_{p}$ the plasma frequency and the $1 / \sqrt{3}$-factor caused by the shape of the sphere. This is the dipole-term. The larger the size of the sphere the more resonances around the $\omega_{p} / \sqrt{3}$-peak appear (if $k a>1$ ) reflecting the surface-mode resonances or higher multipoles, see [3].

The nonlinear interaction of light with small particles was recently studied by Östling et al. [4] using a simplified model to obtain the intensities in second and third harmonic 
generation, SHG and THG, respectively. The calculations yield an enhancement of the total intensities by a factor 5000 in SHG and 200000 in THG in comparison to a plane metal surface for spheres with sizes $a>\lambda_{p} / 2$ in a wide frequency range. Here, $\lambda_{p}$ is the wavelength corresponding to $\omega_{p}$ using the relation $c=\lambda \cdot \omega / 2 \pi$ with $c$ the speed of light in vacuum. This holds especially if the diameter of the sphere roughly equals $\lambda_{p}$.

The correlation between the scattering behavior and the properties of the sphere enables us to obtain the size and complex refractive index of the sphere and in the case of an ensemble of spheres even the size distribution from the scattered intensities. It is a goal of our investigation to show that this is improved by higher harmonics. In this paper we extend the theory of ref. [4] to obtain the angular dependence of the radiated second and third harmonic intensities, compare the linear and nonlinear results examining their characteristic features in higher harmonics. In particular the angular resolved intensities, the degree of polarization of the scattered SHG and THG yield are investigated. In addition, we thoroughly study parameter dependences of the ratio of forward and backward scattering and analyze the origin of the resonance structures.

Our main results are an enhanced size sensitivity of the higher harmonics compared to linear Mie-scattering, thus pronouncing the mentioned characteristic features of the linear Mie-effect in higher harmonic scattering. Similarities between the linear scattering and THG reflect that the scattering is dominated by the same multipoles in both cases. In contrast, different multipoles contribute to SHG resulting in distinct changes of the angular dependence. Because of the more pronounced forward scattering enhanced backward scattering vanishes in the higher harmonics.

The following section II contains the appropriate theoretical calculations. In section III we present the numerical results starting with polar plots of the intensities in SHG and THG, plots for the degree of polarization, the ratio of forward and backward scattering and the integrated intensities as a function of the size of the spheres. In section IV we will give a summary of the results. 


\section{THEORY}

First, we use Mie theory to calculate the linear scattering and the radial component of the polarization $\sigma(\theta, \phi)$ at the surface of the sphere. Then, we determine the radiated intensities in second and third harmonic by matching the electromagnetic fields and the $n$-th power of $\sigma(\theta, \phi)$ with regard to the appropriate boundary conditions, as described by Östling et al. [4]. In section II.D the characteristic quantities of the radiation are determined. These quantities can be compared with experiments.

\section{A. Linear scattering by spheres}

According to the spherical symmetry of the problem we expand the fields in the form

$$
\begin{aligned}
\mathbf{E}^{i}(\mathbf{x})=\sum_{l, m} C(l)\left[a_{M}^{i}(l, m) f_{l}^{i}\left(k_{1} r\right) \mathbf{X}_{l, m}(\theta, \phi)\right. & \\
& \left.+\frac{m}{|m|} a_{E}^{i}(l, m) \frac{1}{\epsilon(\omega) k} \nabla \times f_{l}^{i}\left(k_{1} r\right) \mathbf{X}_{l, m}(\theta, \phi)\right]
\end{aligned}
$$

Therein, $\mathbf{X}_{l, m}$ is a vector spherical harmonic as introduced by Jackson [5] with $C(l)=$

$i^{l} \sqrt{4 \pi(2 l+1)}, k=\omega / c$ and $k_{1}=\sqrt{\epsilon(\omega)} k$. The multipole coefficients $a_{M}^{i}(l, m)$ and $a_{E}^{i}(l, m)$ refer to the magnetic (transverse electric) and electric (transverse magnetic) multipoles. The index $i$ specifies the incident $(i \equiv i n c)$, the scattered $(i \equiv s c)$ or the internal $(i \equiv i n)$ fields. For incident waves of positive and negative helicity we have $a_{M}^{i n c}(l, \pm 1)=a_{E}^{i n c}(l, \pm 1)=1$. In this paper we use a superposition of both to give linear polarization. The spherical Hankel functions $f_{l}^{s c}(k r)=h_{l}^{(1)}(k r)$ and Bessel functions $f_{l}^{i n c, i n}(k r)=j_{l}(k r)$ describe the radial part of the field outside and inside the sphere. The magnetic field is given by the Maxwell-equation for harmonic fields

$$
\mathbf{B}=-i \omega / c \cdot \nabla \times \mathbf{E} .
$$

Using the boundary conditions at the surface of the sphere 


$$
\mathbf{n} \times\left(\mathbf{E}^{s c}+\mathbf{E}^{i n c}\right)=\mathbf{n} \times \mathbf{E}^{i n}
$$

and

$$
\mathbf{n} \times\left(\mathbf{B}^{s c}+\mathbf{B}^{i n c}\right)=\mathbf{n} \times \mathbf{B}^{i n}
$$

we obtain the expansion coefficients of the scattered wave

$$
\begin{aligned}
& a_{E}^{s c}(l, \pm 1)=\left.\frac{j_{l}(k r) \frac{\partial}{\partial r}\left[r j_{l}\left(k_{1} r\right)\right]-\epsilon(\omega) j_{l}\left(k_{1} r\right) \frac{\partial}{\partial r}\left[r j_{l}(k r)\right]}{\epsilon(\omega) j_{l}\left(k_{1} r\right) \frac{\partial}{\partial r}\left[r h_{l}^{(1)}(k r)\right]-h_{l}^{(1)}(k r) \frac{\partial}{\partial r}\left[r j_{l}\left(k_{1} r\right)\right]}\right|_{r=a}, \\
& a_{M}^{s c}(l, \pm 1)=\left.\frac{j_{l}(k r) \frac{\partial}{\partial r}\left[r j_{l}\left(k_{1} r\right)\right]-j_{l}\left(k_{1} r\right) \frac{\partial}{\partial r}\left[r j_{l}(k r)\right]}{j_{l}\left(k_{1} r\right) \frac{\partial}{\partial r}\left[r h_{l}^{(1)}(k r)\right]-h_{l}^{(1)}(k r) \frac{\partial}{\partial r}\left[r j_{l}\left(k_{1} r\right)\right]}\right|_{r=a}
\end{aligned}
$$

at the surface of the sphere with radius $a$. From the continuity of the electrical displacement at the surface of a perfect conductor

$$
\mathbf{n} \cdot\left(\mathbf{D}^{s c}+\mathbf{D}^{i n c}\right)=\mathbf{n} \cdot \mathbf{D}^{i n}
$$

the surface charge results as

$$
\sigma(\theta, \phi)=\frac{1}{4 \pi} R e\left[\left(\mathbf{E}^{s c}+\mathbf{E}^{i n c}-\mathbf{E}^{i n}\right) \cdot \mathbf{n}\right] e^{-i \omega t}
$$

where $\mathbf{n}=\mathbf{r} /|\mathbf{r}|$ and $R e$ denots the real part. Furthermore we expand $\sigma(\theta, \phi)$ in spherical harmonics

$$
\sigma(\theta, \phi)=\frac{1}{2} \sum_{l, m= \pm 1} a_{l, m}^{(1)} Y_{l, m}(\theta, \phi) e^{-i \omega t}+c . c .
$$

The expansion coefficients result from the orthogonality of the spherical harmonics as

$$
a_{l, \pm 1}^{(1)}=\frac{1}{4 \pi}\left(1-\frac{1}{\epsilon(\omega)}\right) \frac{C(l) i \sqrt{l(l+1)}}{k a}\left(j_{l}(k a)+a_{E}^{s c}(l, \pm 1) h_{l}^{(1)}(k a)\right)
$$

\section{B. Sources of the higher harmonic radiation}

In analogy to the linear case we expand the n-th power of the surface charge $\sigma$ in terms of spherical harmonics: 


$$
\sigma^{n}(\theta, \phi)=\frac{1}{2} \sum_{l, m} a_{l, m}^{(n)} Y_{l, m}(\theta, \phi) e^{-n i \omega t}+c . c .
$$

Neglecting time-independent terms we obtain the coefficients in the case of second harmonic generation as

$$
\begin{aligned}
a_{l, 2}^{(2)} & =\frac{1}{2} \sum_{l_{1}=1}^{\infty} \sum_{l_{2}=1}^{\infty} a_{l_{1}, 1}^{(1)} a_{l_{2}, 1}^{(1)} \int Y_{l, 2}^{*} Y_{l_{1}, 1} Y_{l_{2}, 1} d \Omega \\
a_{l,-2}^{(2)} & =\frac{1}{2} \sum_{l_{1}=1}^{\infty} \sum_{l_{2}=1}^{\infty} a_{l_{1},-1}^{(1)} a_{l_{2},-1}^{(1)} \int Y_{l,-2}^{*} Y_{l_{1},-1} Y_{l_{2},-1} d \Omega, \\
a_{l, 0}^{(2)} & =\frac{1}{2} \sum_{l_{1}=1}^{\infty} \sum_{l_{2}=1}^{\infty} a_{l_{1}, 1}^{(1)} a_{l_{2},-1}^{(1)} \int Y_{l, 0}^{*} Y_{l_{1}, 1} Y_{l_{2},-1} d \Omega,
\end{aligned}
$$

and for third harmonic generation (also neglecting terms with $e^{-i \omega t}$ )

$$
\begin{aligned}
a_{l, 1}^{(3)} & =\frac{1}{2} \sum_{l_{1}=1}^{\infty} \sum_{l_{2}=1}^{\infty} a_{l_{1},-1}^{(1)} a_{l_{2},-2}^{(2)} \int Y_{l, 1}^{*} Y_{l_{1}, 1} Y_{l_{2}, 2} d \Omega, \\
a_{l,-1}^{(3)} & =\frac{1}{2} \sum_{l_{1}=1}^{\infty} \sum_{l_{2}=1}^{\infty} a_{l_{1}, 1}^{(1)} a_{l_{2},-2}^{(2)} \int Y_{l,-1}^{*} Y_{l_{1}, 1} Y_{l_{2},-2} d \Omega, \\
a_{l, 3}^{(3)} & =\frac{1}{2} \sum_{l_{1}=1}^{\infty} \sum_{l_{2}=1}^{\infty} a_{l_{1},-1}^{(1)} a_{l_{2}, 2}^{(2)} \int Y_{l, 3}^{*} Y_{l_{1}, 1} Y_{l_{2}, 2} d \Omega, \\
a_{l,-3}^{(3)} & =\frac{1}{2} \sum_{l_{1}=1}^{\infty} \sum_{l_{2}=1}^{\infty} a_{l_{1},-1}^{(1)} a_{l_{2}, 2}^{(2)} \int Y_{l,-3}^{*} Y_{l_{1},-1} Y_{l_{2},-2} d \Omega .
\end{aligned}
$$

The integrals can be expressed by the 3j-symbols and yield the coupling of the multipoles. Because of conservation of angular momentum, only coefficients with $m=0, \pm 2$ in SHG and $m= \pm 1, \pm 3$ in THG differ from zero.

\section{Higher harmonic radiated fields}

In the case of higher harmonics the electric and magnetic fields inside and outside the sphere and the sources are matched by the boundary conditions

$$
\mathbf{n} \cdot\left(\mathbf{D}^{\text {out }}-\mathbf{D}^{\text {in }}\right)=4 \pi \sigma^{n}(\theta, \phi)
$$

and

$$
\mathbf{n} \times\left(\mathbf{E}^{\text {out }}-\mathbf{E}^{\text {in }}\right)=0
$$


As a result of spherical symmetry, only transverse magnetic waves are generated by the oscillating surface charge. Thus the fields in the nonlinear case are

$$
\mathbf{E}^{i}(\theta, \phi)=\sum_{l, m} \frac{m}{|m|} A_{E}^{(n)}(l, m) \frac{1}{\epsilon(n \omega) k} \nabla \times f_{l}^{i}\left(k_{1} r\right) \mathbf{X}_{l, m}(\theta, \phi)
$$

where $i \equiv$ out or $i \equiv i n$, respectively, and $k=n \omega / c, k_{1}=\sqrt{\epsilon(n \omega)} k, f_{l}^{i n}(k r)=j_{l}\left(k_{1} r\right)$ and $f_{l}^{\text {out }}=h_{l}^{(1)}(k r)$. The boundary conditions give the coefficients of the radiated field

$$
A_{E}^{(n)}(l, m)=\frac{\frac{\partial}{\partial r}\left[r j_{l}\left(k_{1} r\right)\right]}{\epsilon(n \omega) j_{l}\left(k_{1} r\right) \frac{\partial}{\partial r}\left[r h_{l}^{(1)}(k r)\right]-h_{l}^{(1)}(k r) \frac{\partial}{\partial r}\left[r j_{l}\left(k_{1} r\right)\right]} \frac{\pi k a}{\sqrt{l(l+1)}} a_{l, m}^{(n)} .
$$

In this case we have $k_{1}=\sqrt{\epsilon(n \omega)} k$.

\section{Calculation of quantities characterizing the radiation}

To study the angular dependence of the scattered field we use the quantity $\left|E_{\phi}(\theta, \phi)\right|^{2}+$ $\left|E_{\theta}(\theta, \phi)\right|^{2}$ according to Born and Wolf [6], where $E_{\theta}(\theta, \phi)$ and $E_{\phi}(\theta, \phi)$ are the tangential components of $\mathbf{E}^{s c}(\theta, \phi)$ in the linear case and of $\mathbf{E}^{\text {out }}(\theta, \phi)$ in the nonlinear case. This definition is equivalent to the absolute value of the radial part of the Poynting vector $|\mathbf{n} \cdot(\mathbf{E} \times \mathbf{H})|$. The following formulas represent $\left|E_{\phi}(\theta, \phi)\right|^{2}$ and $\left|E_{\theta}(\theta, \phi)\right|^{2}$ in the far field approximation. We obtain after evaluating the $m$-summation in the linear case

$$
\begin{aligned}
\left|E_{\phi}(\theta, \phi)\right|^{2} & =\left|\sum_{l=1}^{\infty} C(l)\left(\frac{d P_{l}^{1}(\cos \theta)}{d \theta} a_{M}^{s c}(l, 1)+\frac{P_{l}^{1}(\cos \theta)}{\sin \theta} a_{E}^{s c}(l, 1)\right)\right|^{2} \cdot \sin ^{2} \phi, \\
\left|E_{\theta}(\theta, \phi)\right|^{2} & =\left|\sum_{l=1}^{\infty} C(l)\left(\frac{P_{l}^{1}(\cos \theta)}{\sin \theta} a_{M}^{s c}(l, 1)+\frac{d P_{l}^{1}(\cos \theta)}{d \theta} a_{E}^{s c}(l, 1)\right)\right|^{2} \cdot \cos ^{2} \phi,
\end{aligned}
$$

for second harmonic generation

$$
\begin{aligned}
\left|E_{\phi}(\theta, \phi)\right|^{2}= & \mid \sum_{l=1}^{\infty} \sqrt{4 \pi(2 l+1)}\left(\frac{d P_{l}^{0}(\cos \theta)}{d \theta} A_{E}^{(2)}(l, 0)\right. \\
& \left.+\frac{d P_{l}^{2}(\cos \theta)}{d \theta} 2 K(l) A_{E}^{(2)}(l, 2) \cos (2 \phi)\right)\left.\right|^{2} \\
\left|E_{\theta}(\theta, \phi)\right|^{2} & =\left|\sum_{l=2}^{\infty} \sqrt{4 \pi(2 l+1)} \frac{d P_{l}^{2}(\cos \theta)}{d \theta} A_{E}^{(2)}(l, 2)\right|^{2} \cdot \sin ^{2}(2 \phi)
\end{aligned}
$$

and third harmonic generation 


$$
\begin{aligned}
\left|E_{\phi}(\theta, \phi)\right|^{2}= & \mid \sum_{l=1}^{\infty} \sqrt{4 \pi(2 l+1)}\left(\frac{P_{l}^{1}(\cos \theta)}{\sin \theta} A_{E}^{(3)}(l, 1) \sin \phi\right. \\
& \left.+\frac{P_{l}^{3}(\cos \theta)}{\sin \theta} K(l) A_{E}^{(3)}(l, 3) \sin (3 \phi)\right)\left.\right|^{2} \\
\left|E_{\theta}(\theta, \phi)\right|^{2}= & \mid \sum_{l=1}^{\infty} \sqrt{4 \pi(2 l+1)}\left(\frac{d P_{l}^{1}(\cos \theta)}{d \theta} A_{E}^{(3)}(l, 1) \cos \phi\right. \\
& \left.+\frac{d P_{l}^{3}(\cos \theta)}{d \theta} K(l) A_{E}^{(3)}(l, 3) \cos (3 \phi)\right)\left.\right|^{2}
\end{aligned}
$$

where $K(l)$ are $l$ - and $m$-dependent factors.

Note that the Legendre polynomials $P_{l}^{m}$ with $m=0,2$ are identically zero for $\theta=0, \pi$, since there is no direct scattering in forward nor backward direction in the second harmonic case. Furthermore the $\phi$ - dependence of the linear scattering and THG is described by the interval $(0, \pi)$ and by $\left(0, \frac{\pi}{2}\right)$ in SHG according to the symmetries of the sine and cosine terms.

We will plot the degree of polarization defined as

$$
P(\theta)=\frac{I_{\|}-I_{\perp}}{I_{\|}+I_{\perp}},
$$

with

$$
I_{\|}=\left|E_{\theta}\left(\theta, \phi=\frac{\pi}{2}\right)\right|^{2}+\left|E_{\phi}\left(\theta, \phi=\frac{\pi}{2}\right)\right|^{2}
$$

and

$$
I_{\perp}=\left|E_{\theta}(\theta, \phi=0)\right|^{2}+\left|E_{\phi}(\theta, \phi=0)\right|^{2} .
$$

To measure the asymmetry of forward and backward scattering in the Mie-range we introduce the quantity

$$
R=\frac{I_{\text {forw }}-I_{\text {back }}}{I_{\text {forw }}+I_{\text {back }}}
$$

which we call the "degree of Mie-asymmetry". In the linear case $I_{\text {forw }}$ and $I_{b a c k}$ are the scattering intensities taken at $\theta=0$ and $\theta=\pi$ respectively. As these quantities are identically 
zero in SHG, we use for $I_{\text {forw }}$ and $I_{\text {back }}$ the maxima of the scattering intensities along the direction of propagation of the incident wave for $\phi=0, \frac{\pi}{4}, \frac{\pi}{2}$ and $\theta$ covering the interval $(0, \pi)$. In THG the angular dependence fo the radiated intensities is more complicated compared to the linear case and we take the maximum for $\phi=0$ and $\theta$ ranging from 0 to $\pi$.

Finally, we calculate the angle-integrated scattered intensities. We obtain in the linear case

$$
Q_{s c}^{(1)}=\frac{1}{\pi(k a)^{2}} \sum_{l, m} \frac{2 l+1}{l(l+1)}\left(\left|a_{E}^{s c}(l, m)\right|^{2}+\left|a_{M}^{s c}(l, m)\right|^{2}\right)
$$

and for the $n$-th harmonic

$$
Q_{s c}^{(n)}=\frac{1}{\pi(k a)^{2}} \sum_{l, m}\left|A_{E}^{(n)}(l, m)\right|^{2}
$$

Here $Q_{s c}^{(n)}$ is in units of the geometric cross section of the sphere $\pi a^{2}$. In this formulation the optical theorem, which links the extinction efficiency to the normalized scattering amplitude in forward direction, has the form

$$
Q_{e x t}=\frac{2}{\pi(k a)^{2}} \cdot\left(\left|E_{\theta}(\theta=0)\right|^{2}+\left|E_{\phi}(\theta=0)\right|^{2}\right)
$$

with

$$
Q_{e x t}=\frac{1}{\pi(k a)^{2}} \sum_{l, m}(2 l+1)\left\{\operatorname{Re}\left[a_{E}^{s c}(l, m)\right]+\operatorname{Re}\left[a_{M}^{s c}(l, m)\right]\right\}
$$




\section{NUMERICAL RESULTS}

We present numerical results for the angular dependence of the radiated intensities obtained using Eqs. (17)-(19). The degree of polarization characterizing the radiation, for example its angular dependence. The degree of Mie-asymmetry $R$ calculated using Eq.(23) gives us the strength of the asymmetry along the direction of propagation according to the Mie-effect. The integrated intensities give us the resonances as a funtion of cluster size. In all cases we compare the linear results with the results of second and third harmonic generation as a function of the size parameter $k a$ and the material-properties, referring to iron and nickel at a fixed optical wavelength of $617 \mathrm{~nm}$. To check the numerical accuracy we compare the linear results with those of ref. [2]. We find excellent agreement. Furthermore, we check

the optical theorem. By determining $\Delta Q \equiv\left|Q_{e x t}-2 \cdot\left(\left|E_{\theta}\right|^{2}+\left|E_{\phi}\right|^{2}\right)_{\theta=0} /\left[\pi(k a)^{2}\right]\right|$ as a function of $k a$ we find that this is satisfied to an accuarcy better than $10^{-12}$.

As input parameter we take the complex refractive indices measured by Johnson and Christy [7]. In the linear case, we also use for comparison with other calculations the refractive index of water droplets, as given by Bohren and Huffman [2]. These values are listed in Tab. I. The refractive index is constant in all figures unless something else is specified. Thus varying the size parameter $k a$ means varying the size of the sphere.

\section{A. Angular dependence of the intensities}

First, we show polar plots of $\left|E_{\phi}\right|^{2}+\left|E_{\theta}\right|^{2}$ (see Figs. 3 - 5). In general, the shape of the plots is governed by the values of the coefficients in the series expansions Eqs. (17)-(19). It is well known [5] that in the linear series (17) only terms with $l \leq k a$ contribute significantly. For $l>k a$ the terms decrease very rapidly, whereas for $l \ll k a$ they have comparable magnitudes. We restrict our calculation to $l<\left[\operatorname{Max}\left(\tilde{n} \cdot k a, \tilde{n}^{\prime} \cdot k a\right)+15\right]$ with a maximum value of $l$ of 50 where $\tilde{n}$ and $\tilde{n}^{\prime}$ are the real and imaginary parts of the complex refractive index. This gives satisfactory convergence of the series up to $k a<10$ in all harmonics. 
To qualify the angular dependence the range of size parameters up to 5 is sufficient. In this range the $l$-values of the dominating terms are a little smaller than $k a$ and no terms with $l \ll k a$ exist. Terms with $l \leq k a$ can be very different from each other in contrast to terms with $l \ll k a$ which have comparable magnitudes. Thus, a pronounced transition range from pure dipole-scattering to pure Mie-scattering exists. Of course we cannot reach the transition from Mie-scattering to the optical limit of reflection, due to the numerical limit of a maximum value of $l$ of 50 .

The geometry of the scattering is specified in the inset of Fig. 3 with the direction of propagation of the incident wave being parallel to the positive y-axis and polarization along the positive z-axis. Fig. 3 (a) shows Rayleigh-scattering according to the dipole-term with $l=1$. The characteristic $\cos ^{2} \theta$-dependence appears along the $\mathrm{x}$-z-plane. The other plots for linear optics (Figs. 3) show the well known results [1, 2,6]. For a value of $k a=1$ asymmetry of forward and backward scattering appears according to the Mie-effect. The ratio of forward to backward scattering $I_{\text {forw }} / I_{\text {back }}$ increases strongly with increasing size parameter $k a$ beginning at a value of $k a \approx 1$. "New" maxima grow out in the backward direction and move to the forward direction with increasing $k a$. The $\phi$-dependence is not as striking and not as complicated as the $\theta$-dependence since we have a superposition of the form $A(\theta) \cdot \cos ^{2} \phi+B(\theta) \cdot \sin ^{2} \phi$, the scattering-behavior is dominated by the strong increase in forward scattering described by the $\theta$-dependence. The fact that the $\phi$ - dependence is described by the intervall $(0, \pi)$ is most important for the linear case and harmonics with odd order and differs from SHG and harmonics or even order where the $\phi$-dependence is fully described by the intervall $\left(0, \frac{\pi}{2}\right)$.

The polar-plots in the case of THG are quite similar to the linear case up to values of $k a \approx 2$. The differences between Figs. 3 (b) and 1 (b) with $k a=1$ reflect the stronger increase in the ratio of forward to backward scattering in THG with increasing $k a$. The plots in Figs. 3 (c) and 1 (c) with $k a=2$ are very similar apart from one more maximum appearing in the third harmonic case for $\theta \approx f r a c \pi 2$. For $k a=5$ the intensities parallel to the direction of polarization are much larger in third harmonic than in the linear case. 
Note, the different scales of the axes for different $k a$. The terms for $m=3$ in THG are negligble compared to the terms with $m=1$. So the differences in the magnitudes of the linear and third harmonic intensities are caused by the coefficients $a_{E}^{s c}(l, 1), a_{M}^{s c}(l, 1)$ and $A_{E}^{(3)}(l, 1)$ only.

The angular dependence of the intensities in second harmonic is very different from the linear and third harmonic cases. The disappearing direct forward and backward intensities and the $\cos ^{2}(2 \phi)$ and $\sin ^{2}(2 \phi)$ behavior produce the club-shaped structure, which is shown in Figure 5. But the main features of the linear and third harmonic plots appear also in second harmonic. The plots become asymetrical with respect to $\theta$ in the range of $k a \approx 1$. The ratio of forward to backward scattering increases with $k a$ and is between the linear and third harmonic one. Values of the forward to backward scattering ratio with different $k a$-values are listed in Tab. II. In general harmonics with even order will show an angular dependence like SHG, because the Legendre polynomials of $m \neq 1$ vanish at $\theta=0, \pi$ and $\phi$ will appear in the cosine and sine-terms in connection with $n=0,2, ., 2 p$ ( $p$ integer). Analogous harmonics with odd order will behave similar to the linear case.

\section{B. Polarization}

To learn more about the angular dependence, especially the $k a$-dependence of the intensity maxima, we calculate the degree of polarization using Eq. (20). First, we plot the polarization in the case of Rayleigh-scattering (Fig. 6 (a)). For convenience and in agreement with the preceding section each of the figures in Fig. 6 contain the linear and third harmonic curves. The plots of Rayleigh- scattering (Fig. 6 (a)) are identical. With increasing size parameter the maximum of the polarization moves to smaller angles (the forward direction) in agreement with the intensity maxima. In third harmonic generation they move faster than in the linear case (Fig. 6 (b)). For $k a=2$ (Fig. 6 (c)) there is one more maximum in THG in agreement with the Figs. 3 (c) and 4 (c). Increasing the size parameter up to 5 destroys the correlation between the peaks in the polarization plots and the polar plots 
in THG. For example the "double" peaks in the third harmonic plot with $k a=5$ cannot be identified with "double" peaks in the polar plots but they reflect that the intensities at $\phi=0$ are comparable to those at $\phi=\frac{\pi}{2}$ in contradiction to the linear case. In the linear case the polarization even for values higher than $k a=5$ reproduces the position of the peaks in the polar plots and is mainly perpendicular $(P(\theta)>0)$. This is an artifact of the chosen refractive index and is not characteristic for linear Mie- scattering in general. For imaginary parts of the refractive index close to zero, the polarization is mainly perpendicular.

The polarization plots in second harmonic generation reflect the different shape of the polar plots. Since the $P(\theta=0)$ and $P(\theta=\pi)$ values do not exist and the limits $\lim _{\theta \rightarrow 0} P(\theta)$ and $\lim _{\theta \rightarrow \pi} P(\theta)$ are different, the plot in the Rayleigh-range is asymmetric. The decrease of $|P|$ up to zero with increasing $k a$ is characteristic for SHG. The square of the absolute value of the $\theta$ - component of the electric field $\left|E_{\theta}\right|^{2}$ is identically zero for $\phi=0, \pi$. Thus $P(\theta)$ is a measure of the importance of the $(m=2)$-term in $\left|E_{\phi}(\theta, \phi)\right|^{2}$ with respect to the $(m=0)$-term. Thus the $(m=0)$-term can be neglected with increasing $k a$. Only for very small $k a$, the structure in the polarization plots correlates with the shape of the polar plots. The first "new" maximum appears as zero in the polarization. For larger $k a$, however, there is no correlation any more. 


\section{Forward vs. backward scattering}

By computing $R$ defined in Eq. (23) which is a measure of the difference between the forward and backward intensities as a function of the size parameter $k a$ and the real or imaginary part of the refractive index $N=\tilde{n}+i \cdot \tilde{n}^{\prime}$, we want to study the development of the asymmetry of forward to backward intensities. Of particular interest will be enhanced backward scattering.

Figure 8 (a) shows the increase of forward scattering with the size of the sphere in linear scattering. The oscillations correlate with intensity maxima in the backward direction resulting from maxima of the coefficients, see Probert-Jones [8]. Enhanced backward scattering appears only in the small range of $0.5<k a<1$ in the case of iron. The curve for water droplets shows no enhanced backscattering, but the overall behavior is the same as for metals. Varying the imaginary part of the refractive index diminishes the forward scattering. In the case of $k a=1$ and $\tilde{n}^{\prime}>5$ an enhancement of the backward scattering occurs. The $\tilde{n}$ dependence of $R$ is similar.

In the higher harmonic case the Mie-effect is strongly enhanced. The limit of one is obtained earlier than in the linear case. The higher the order, the stronger is the enhancement of the forward scattering. Regions of enhanced backward scattering are hard to find. In the case of second harmonic generation, they exist only for small $\tilde{n}$ or $\tilde{n}^{\prime}$ around 5 and small

$k a$ whereas we could not find enhanced backward scattering for metal- clusters $\left(\tilde{n}^{\prime} \gg 0\right)$ in third harmonic generation so far. 


\section{Integrated intensities}

Mie-Resonances appear with increasing radius $a$ of the sphere or absolute value of the wave vector $k$ of the incident light. In this section we will only deal with the size-dependent resonances.

In Fig. 1 we show the scattering efficiency $Q_{s c}$ of water droplets as a function of the size parameter. The main features are the interference structure built up by interferences between the incident wave and forward scattered light and the ripple structure reflecting resonant surface modes. Furthermore, the results suggest the optical paradoxon $\lim _{k a \rightarrow \infty} Q_{e x t}(k a)=$

2. The ripple structure correlates with resonances in the coefficients $a_{E}^{s c}(l, m)$ and $a_{M}^{s c}(l, m)$. They are resonant if their imaginary part is zero. Fig. 12 shows the first resonance of $a_{E}^{s c}(13,1)$ for a real refractive index with $\tilde{n}=1.5$ and $\tilde{n}^{\prime}=0$. The resonance of $a_{M}^{s c}(13,1)$ occurs for $k a \approx 11$ (see Chýlek [9]). For large size parameters the distance of the ripples can be expressed directly by the refractive index. Finite imaginary parts of the refractive index would damp the resonance. Then the values of the coefficients at the resonant point would be smaller than 1 . Even if we take $\tilde{n}^{\prime} \approx 0.1$ the ripple structure in Fig. 12 does not appear. Accordingly, there is no ripple structure in the scattering efficency of $\mathrm{Fe}$ and $\mathrm{Ni}$ as a function of size as shown in Fig. 13 (a).

In higher harmonics no structure correlated with resonances of the coefficient can be detected even for vanishing imaginary part of the refractive index up to a numerical accuracy of $10^{-10}$. Each coefficient $a_{l, m}^{(n)}$ is a combination of all linear electric multipole coefficients determined by Eqs. (11) and (12), respectively. Correspondingly, the size dependence of the coefficients $A_{E}^{(n)}(l, m)$ has many small peaks (Fig. 12 (b) and (c)). In contrast, the size resonances caused by multipole-combinations, and known as interference structure in linear scattering, are in the case of metals more pronounced in SHG (compare Fig. 13 (a) and (b)). In THG (Fig. 13 (c)) the first peak is even more dominant and the resonances are visible only for enhanced resolution. In both cases, the position of the resonances has a weak dependence on the refractive index, but the number of peaks in SHG is twice the peak- 
number in THG if $0<k a<10$. Decreasing the imaginary part of the refractive index down to zero changes the behavior drastically. Instead of a dominating first peak the scattering efficiency now shows an continuous increase for $0<k a<20$ with oscillations stronger than in the case of $\tilde{n}^{\prime}>1$. In all three cases the absolute values grow with the absolut value of the refractive index if metals are considered. 


\section{SUMMARY}

By extending the classical model introduced by Östling et al. [?] we calculated the angular dependence of the second and third harmonic intensities radiated by spherical metal clusters. Therein the n-th power of the surface charge density induced by linear polarized light is used as the source of the fields radiated in higher harmonics [10]. The source represents the discontinuity of the electrical displacement at the surface of the sphere.

We find that the forward Mie-scattering is in the nonlinear case even more strongly enhanced (see Table II). The nonlinear optical response yields a stronger size sensitivity. Higher multipoles contribute already at smaller size parameters $k a$. For example, we find that the light intensities perpendicular to the forward direction divided by the light intensities in forward direction is much larger in the third harmonic than in the linear case. Since the values of the Legendre polynomials with azimuthal quantum numbers $m=0, \pm 2$ vanish for $\theta=0, \pi$ and due to the $\phi$-dependence of the form $A \cdot \cos ^{2}(2 \phi)+B \cdot \sin ^{2}(2 \phi)$, see Eq. (18), the angular dependence of the second harmonic intensities is very different from the linear and third harmonic distributions. Especially, direct forward and backward scattering vanishes. In contrast, the linear Mie-scattering results (terms with $m= \pm 1$ only) and THG (terms with $m= \pm 1, \pm 3$ ) are similar. In particular the terms with $m= \pm 3$ in THG and $m=0$ in SHG can be neglected, since their absolute values are much smaller than the other contributions. The more pronounced Mie-effect prevents enhanced backward scattering in higher harmonics (Figs. 9).

In order to compare with our theory an experimental investigation of the nonlinear Mie-scattering would be interesting. Mie-resonances play an important role in the field of

photonic bandstructure in high refractive materials (see John [11]), but they are studied so far in the linear case only. Also applications of the theory to the study of fullerenes and problems in biophysics (detection and growth modes of tumor cells) would be interesting.

We will extend the theory to ellipsoidal objects to solve the classical problem of a one to one correspondence of scattering profile and particle shape. Furthermore, the expected 
curvature sensitivity of the nonlinear optical response even to particles with sizes much smaller than the wavelength, should be detectable in the nonlinear scattering. Thus, the higher harmonics are a particularly useful probe for detecting small particle sizes and shapes. 


\section{FIGURES}

FIG. 1. The linear extinction $Q_{e x t}(k a)$ as a function of the size parameter $k a$ and varying the radius of the sphere for water droplets with complex refractive index $N=1.33+i \cdot 10^{-7}$.

FIG. 2. Difference $\Delta Q=Q_{e x t}-2 \cdot\left(\left|E_{\theta}\right|^{2}+\left|E_{\phi}\right|^{2}\right)_{\theta=0} /\left[\pi(k a)^{2}\right]$ of the extinction efficiency and the modified forward scattering amplitude, as a function of the size parameter $k a$. This illustrates how our numerical calculations fulfill the optical theorem.

FIG. 3. Polar plots of the linear scattered intensities for Fe: (a) scattered linear intensity $I_{\omega}(\theta, \phi)$ in units of $10^{-12}$ with $k a=0.001$, (b) $I_{\omega}(\theta, \phi)$ in units of $10^{-4}$ with $k a=1$, (c) $I_{\omega}(\theta, \phi)$ in units of 1 with $k a=2$, and (d) $I_{\omega}(\theta, \phi)$ with $k a=5$ in units of 1 . The inset shows the scattering geometry. The direction of the incident light is determined by $\theta=0$ with polarization along the axis defined by $\phi=0$ and $\theta=\pi / 2$.

FIG. 4. Polar plots of the third harmonic (THG) intensities for Fe: (a) scattered $3 \omega$-intensity $I_{3 \omega}(\theta, \phi)$ in umits of $10^{-15}$ with $k a=0.001$, (b) $I_{3 \omega}(\theta, \phi)$ in units of 1 with $k a=1$, (c) $I_{3 \omega}(\theta, \phi)$ in units of $10^{-5}$ with $k a=2$, and (d) $I_{\omega}(\theta, \phi)$ in units of $10^{-5}$ with $k a=5$.

FIG. 5. Polar plots of the second harmonic (SHG) intensities for Fe: (a) scattered $2 \omega$-intensity $I_{2 \omega}(\theta, \phi)$ in units of $10^{-18}$ with $k a=0.001$, (b) $I_{2 \omega}(\theta, \phi)$ in units of 1 with $k a=1$, (c) $I_{2 \omega}(\theta, \phi)$ in units of $10^{2}$ with $k a=2$ and, (d) $I_{\omega}(\theta, \phi)$ in units of $10^{6}$ with $k a=5$.

FIG. 6. Degree of polarization $P$ for the linear and third harmonic case as a function of the polar angle $\theta$ (a) for $k a=0.001$, (b) for $k a=1$., (c) for $k a=2$, and (d) for $k a=5$.

FIG. 7. Degree of polarization $P$ for the second harmonic case as a function of the polar angle $\theta$ (a) for $k a=0.001$, (b) for $k a=1$, (c) for $k a=2$, and (d) for $k a=5$.

FIG. 8. Degree of Mie-asymmetry $R$ (defined as $R=\left(I_{\text {forw }}-I_{\text {back }}\right) /\left(I_{\text {forw }}+I_{\text {back }}\right)$, see Eq.(23)) in the linear case (a) as a function of the size parameter $k a$ for refractive indices referring to $\mathrm{Fe}, \mathrm{Ni}$ and water droplets, (b) as a function of the real part of the refractive index $\tilde{n}$, and (c) as a function of the imaginary part of the refractive index $\tilde{n}^{\prime}$ for size paramters $k a=1$ and $k a=5$ using values of $\tilde{n}, \tilde{n}^{\prime}$ for Fe and Ni. 
FIG. 9. Degree of Mie-asymmetry $R$ as a function of the size parameter $k a$ for (a) SHG, and (b) THG with the refractive indices referring to Fe and Ni, respectively.

FIG. 10. Degree of Mie-asymmetry $R$ as a function of the real part of the refractive index $\tilde{n}$. For $k a=1$ and $k a=5$ for (a) SHG, and (b) THG. $\tilde{n}^{\prime}$ corresponds to Fe and Ni.

FIG. 11. Degree of Mie-asymmetry $R$ as a function of the imaginary part of the refractive index $\tilde{n}^{\prime}$. For $k a=1$ and $k a=5$ for (a) SHG, and (b) THG. $\tilde{n}$ corresponds to Fe and Ni.

FIG. 12. (a) Real and imaginary part of the expansion coefficient of the scattered linear fields $a_{E}^{s c}(l=13, m=1)$ as a function of size parameter $k a$ and for a real refractive index $\tilde{n}=1.5$. The first peak represents the lowest resonance of the multipole mode with $l=13$. The real and imaginary parts of the expansion coefficients of the SHG and THG fields $A_{8,2}^{(2)}$ and $A_{8,1}^{(3)}$, respectively, as a function of the size parameter $k a$ are shown in Figs. (b) and (c). The results in (b) and (c) show that the resonance peaks of the linear coefficients contribute to the higher harmonic coefficients: The maxima and minima of $A^{(2)}$ and $A^{(3)}$ are essentially determined by the resonances of the linear coefficients $a_{E}^{s c}$.

FIG. 13. Scattering efficiencies $Q_{s c}$ as a function of size parameter $k a$ for (a) linear scattering (b) SHG, and (c) THG in the case of Fe and Ni, respectively. 


\section{TABLES}

TABLE I. Ratio of forward to backward intensities as a function of $k a$ in the linear case, SHG and THG.

\begin{tabular}{|c||c|c|c|}
\hline \multicolumn{1}{|c||}{} & \multicolumn{3}{c|}{$I_{\text {forw }} / I_{\text {back }}$} \\
\hline$k a$ & $\operatorname{lin}$ & $\mathrm{SHG}$ & $\mathrm{THG}$ \\
\hline \hline 1 & 1.4 & 2.7 & 15 \\
2.1 & 8.7 & 10 & 113 \\
4.8 & 64 & 136 & 206 \\
\hline
\end{tabular}

TABLE II. Complex refractive indices $N=\tilde{n}+i \tilde{n}^{\prime}$ for iron and nickel used in this work at $\omega, 2 \omega$ and $3 \omega$. The wavelength is $\lambda=617 \mathrm{~nm}$ and $\omega=2 \pi / \lambda \cdot c$.

\begin{tabular}{|c||c|c|c|c|}
\hline \multicolumn{1}{|c||}{} & \multicolumn{2}{c|}{ iron } & \multicolumn{2}{c|}{ nickel } \\
\hline & $\tilde{n}$ & $\tilde{n}^{\prime}$ & $\tilde{n}$ & $\tilde{n}^{\prime}$ \\
\hline \hline$\omega$ & 2.88 & 3.05 & 1.99 & 4.02 \\
$2 \omega$ & 1.69 & 2.06 & 2.01 & 2.18 \\
$3 \omega$ & 1.49 & 1.41 & 1.29 & 1.89 \\
\hline
\end{tabular}




\section{REFERENCES}

[1] G. Mie, Ann. Phys. (Leipzig) 25, 377 (1908)

[2] C. F. Bohren and D. R. Huffman: Absorption and Scattering of Light by Small Particles, (Wiley, New York, 1983)

[3] S. S. Martinos, Phys. Rev. B 31, 2029 (1985)

[4] D. Östling, P. Stampfli and K. H. Bennemann, Z. Phys. D 28, 169-175 (1993)

[5] J. D. Jackson: Classical Electrodynamics (Wiley, New York, 1975)

[6] M. Born and E. Wolf: Principles of Optics, (Pergamon Press, Oxford, 1975)

[7] P. B. Johnson, R. W. Christy, Phys. Rev. B 9, 5056- 5070 (1974)

[8] J. R. Probert-Jones, J. Opt. Soc. Am. A, 1 8, 822 (1984)

[9] P. Chýlek, J. Opt. Soc. Am., 66 3, 285-287 (1976)

[10] In addition the following approximations are made in ref. [?]: (a) The nonlinear susceptibilities are taken to be frequency independent, $\chi^{(n)}(\omega)=$ const., (b) the surface charge is assumed to be $\sigma(n \omega)=c \cdot \sigma^{n}(\omega)$, and (c) the proportionality-factor $c$ has not been determined by Östling et al. since they focus on relative intensities. It would be of interest to compare these approximations with a detailed microscopic theory of the nonlinear susceptibilities $\chi^{(n)}(\omega)$.

[11] S. John, Localization of Light, Physics Today, May 1991 